Int J Microcirc 1997;17:397-398

\title{
Subject Index Vol. 17, 1997
}

Acetylcholine 113 Adhesion molecule 138 Adventitia 280 Analysis of variance 123 Anesthetic depth 385 Angiogenesis 1,75,138,159,231,

314 Aortic aneurysm repair 374 L-Arginine 107

Arterial blood pressure waves 346 Arteriolar diameter 164

- $\quad$ vasomotion 164

Arterioles, third order 113

Atherogenesis 280

Atherosclerosis 280

Autonomic nervous system 385

Autonomous nervous system 346

Basement membrane 257 Basic fibroblast factor 314

- growth factor 1 Blood flow 330

- modulation 346

- sensor 360

pressure rhythmicity 385 Bone 1

Calibration 97 Capillaroscopy 97 Capillary luminal size 93

- permeability 150,241

Cardiovascular monitoring 374

Cartilage 1

Cell adhesion molecules 138,231,257

polarity 223

surface receptors 257 Cellular hypoxia 67 Chaos analysis 374 Chorioallantoic membrane, chick 75 Cold 55

Collar model 280 Coronary anatomy 337

capillaries 29 Cremaster flaps 113 Cutaneous arterioles, mouse 61

microcirculation 346, 385 Cyclooxygenase products 41 Cytokine sequestration 257 Cytoskeletal proteins 257

Development 337 Diabetes mellitus 190

Digital image processing 97

- $\quad$ picture processing 322

Dil-Ac-LDL 159

Diltiazem 164

Dimension 97

Dorsal skin fold chamber, mouse 328

Drugs 15

Dynamic capillaroscopy 184

Edema 67

Eigenoscillations 360 Ellipsometry 257, 360 Endothelial calcium 241

cell(s) 67,223 
- senescence 75

- $\quad$ inward rectifying potassium channel

248

Endothelium 41,231,257,273,280 Endurance training 93 Epithelial cells 223 Extracellular matrix 257

- $\quad$ sodium concentration 248

Fast Fourier transform 385 Fentanyl 305 Fibre matrix 241 Flow distribution 337

motion 33,175

peak 248

sensor 248 Fluanisone 305 Fluid exchange 241 Fluorescein 150

angiography 22

isothiocyanate dextran 75 Fluorescence microlymphography 143

videomicroscopy 190 Fractal dimension 374

General anesthesia 385

Gingivitis 346

Ginkgo biloba (EGb 761) extract 61

Hamster cheek pouch 164 Hematocrit 33 Hemodynamics 305 Heparan sulfate proteoglycan 360

Heparin fractions 314 Hepatic microcirculation 48 Histamine 107 Human hearts 29

Hypertension 80, 273

Hypothermia 61 Hypoxia 280

Inflammation 257 Infra-red, near 130 Inspiratory gasp 86 Instrumentation 322 Insulin-dependent diabetes mellitus 150 Interstitial permeability 241

- $\quad$ space, permeability 150

Intestinal blood flow 15

Intraarterial injection 190

Intravital microscopy 305,328

Intrinsic lymphatic contraction 143

Invasive monitoring 374

Isoproterenol 164

Joint 130

Laser Doppler 330

- anemometry 346

- flowmetry 15,48,80,175,298

- fluxmetry $22,55,86,184$

- imager 130

- perfusion monitoring 33

scanning 123 Leukocyte(s) 290

rolling 305

Local oxygen consumption 337 Lymphedema 143

Membrane asymmetry 223 Microangiopathy 150 Microcirculation 41, 80, 113, 138, 159,

164,190,322,328 Microlymphatic backflow 143 Microspheres 130,330 Microvasculature 328

Microvessels 159 Migrating motor complex 15 Monoclonal antibody 138 Monocrotaline 290

Morphometry 29 Motility 15 Myocardium 337 Myogenic response 346

Na-fiuorescein $19024 \mathrm{Na}+$ efflux $360(\mathrm{Na}++\mathrm{K}+)$-ATPase $360 \mathrm{NADH}$ fluorescence 360 Nerve stimulation 48 
KA R.G E R. (C) 1997 S. Karger AG, Basel

Fax+ 41613061234 E-Mail karger@karger.ch www.karger.com

397

Neurogenic vasoconstriction 346 Nitric oxide 107,273 Nitroprusside 113 Nonlinear dynamics 337 Norepinephrine 48, 113

Oscillatory domain 360 Oxygen supply, heart 29

Paracellular pathway 223 Pathophysiology 231 Pentobarbital 305 Perfused liver 48 Perfusion 130

Peripheral vascular disease 184 Permselectivity 75 Phase space plot 374 Phenylephrine 164 Phosphofructokinase 360 Photoplethysmography 346, 385 Phototoxicity 322 Plantaris muscle, rat 93 Platelet(s) 290 - activation 61

Platelet-derived growth factor 280 Portal vascular resistance 48 Prazosin 164

Pulmonary hypertension 290 Pulpal blood flow 298

Rat(s) 175,330 Rauwolscine 164

Raynaud's phenomenon 61 Reactive hyperemia 190

- $\quad$ oxygen species 67

Reactivity 107

Respiration-related flow modulation 346 Revascularization 138

rhG-CSF 10

Self-organization 385 Signal transduction 231 Simulation study 123 Skeletal muscle 175 Skin 55 circulation 22

fold chamber 1,328 -, human 143

microangiopathy 190

microcirculation 184,305

vasomotor reflexes 86 Slow waves 360

Small intestine 15,330 Sodium-cotransport systems 223 Spatial variation 123 Spatiotemporal organization 360 Species specificity 138 Substance P 107 SuffusateP02 113 Sympathetic nervous system 41,385

- $\quad$ tests 80

Synergetics 385

Systemic lupus erythematosus 184

Tension 360 Thromboxane 61 Training intensity 93 Transcellular transport 223 Transplantation 159 Transporter asymmetry 223 Trauma 298 Tumo(u)r angiogenesis 328

cell migration and motility 257

invasion and metastasis 257

Ultrastructural morphometry 75

Vasavasorum 280 Vascular effects 10

endothelial growth factor 280,314

failure 10

smooth muscle 360 Vasoconstriction 55, 346 Vasodilation 55 Vasomotion 15, 175, 346

Vasomotor tone 113 Vasorelaxation 113 Vasospasm 61

Vital capillary microscopy 190

Xenon-133 clearance 22 
Int J Microcirc Vol. 17, 1997

Subject Index 